\title{
CircLARP4 Suppresses Cell Proliferation, Invasion and Glycolysis and Promotes Apoptosis in Non-Small Cell Lung Cancer by Targeting miR-I35b
}

This article was published in the following Dove Press journal: OncoTargets and Therapy

\section{Huawei Lu (D) \\ Qingwei Guo* Guozhang Mao Junwei Zhu \\ Fei Li}

Department of General Thoracic Surgery, Zhoukou Central Hospital, Zhoukou 466000, Henan, People's Republic of China

*These authors contributed equally to this work
Correspondence: Huawei Lu Department of General Thoracic Surgery, Zhoukou Central Hospital, No. 26

Renmin Road, Chuanhui District, Zhoukou 466000, Henan, People's

Republic of China

Tel +863948521601

Email 57885641@qq.com
Background: CircLARP4 is reported to act as a tumor suppressor in some cancers. However, the detailed roles and molecular basis of circLARP4 in non-small cell lung cancer (NSCLC) tumorigenesis are still unclear. The aim of the study is to explore the potential roles and molecular basis of circLARP4 in NSCLC tumorigenesis.

Materials and Methods: qRT-PCR was taken to detect circLARP4 and miR-135b expressions. MTT assay, transwell invasion assay and flow cytometry analysis were applied to evaluate cell proliferation, invasion and apoptosis, respectively. Glycolysis was assessed by measuring hexokinase2 (HK2) expression, glucose consumption and lactate production. Association between circLARP4 and miR-135a was examined by luciferase reporter and RIP assays. The changes of the phosphatase and tension homolog (PTEN)/protein kinase B (AKT)/hypoxia-inducible factor-1 $\alpha$ (HIF-1 $\alpha$ ) pathway were evaluated by Western blot. The nude mouse xenograft models were applied to verify the regulation of circLARP4 in vivo.

Results: CircLARP4 was decreased in NSCLC tissues and cells. CircLARP4 overexpression blocked cell proliferation and invasion, and facilitated apoptosis in NSCLC cells. Meanwhile, circLARP4 overexpression suppressed glycolysis in NSCLC cells, as evidenced by the reduced HK2, glucose consumption and lactate production levels. Further analyses proved a downregulation of miR-135b by circLARP4 in a ceRNA-dependent manner in NSCLC cells. CircLARP4-mediated tumor suppression on NSCLC progression was partially overturned by overexpressing miR-135b. Moreover, we confirmed that circLARP4 had antitumor effect on xenograft tumors and downregulated miR-135b. Furthermore, circLARP4 overexpression inhibited the PTEN/AKT/HIF-1 $\alpha$ pathway in NSCLC cells and xenograft tumors by downregulating miR-135b.

Conclusion: Our findings suggested that circLARP4 suppressed NSCLC progression by sponging miR-135b through inactivation of the PTEN/AKT/HIF-1 $\alpha$ pathway, which broadens our understanding concerning the roles of circLARP4 in NSCLC tumorigenesis.

Keywords: circLARP4, miR-135b, non-small cell lung cancer, glycolysis, HK2, the PTEN/ AKT/HIF-1 $\alpha$ pathway

\section{Introduction}

Lung cancer, belonging to the commonest frequently diagnosed visceral tumors, remains a critical contributor to cancer-related death throughout the world, with an exceptionally high rate of incidence and mortality. ${ }^{1}$ As the major pathological type of lung cancer, non-small cell lung cancer (NSCLC) makes up an estimated $85 \%$ of total of lung cancer and remains a serious health problem worldwide. ${ }^{2}$ However, the 
majority of NSCLC patients are diagnosed at an advanced stage due to unobvious incipient symptoms and lack of specific diagnosis biomarkers for NSCLC. ${ }^{3}$ Despite significant progresses made in the detection and clinical treatment of NSCLC, the therapeutic outcomes are still disappointing and the five-year survival rate of patients suffering from metastatic NSCLC ranges from approximately $4 \%$ to $17 \%$. ${ }^{4}$ Hence, there is a great demand to elucidate the molecular basis of the development of NSCLC, as well as develop novel effective therapeutic interventions.

Circular RNA (circRNA) is a specific type of newly discovered non-coding RNA with limited protein coding potential, which is characterized by the presence of $3^{\prime}-$ poly A tails and a covalently closed continuous loop without $5^{\prime}$ to $3^{\prime}$ polarity. ${ }^{5}$ The research on circRNAs has evoked considerable interest due to their roles as crucial regulators in a wide range of pathophysiological processes, such as cell proliferation, apoptosis, metastasis and cancer progression. ${ }^{6}$ Up to now, widespread evidence has demonstrated that dysregulation of circRNAs is observed in various types of human malignancies and implicated in the carcinogenesis and tumorigenesis of human cancers, including NSCLC. ${ }^{7,8}$ The published studies have shown that circLARP4 is a recently identified circRNA derived from La-related protein 4 (LARP4) gene locus, which is a La-related RNA-binding protein involved in the regulation of cancer cell migration and invasion. ${ }^{9}$ A previous study has demonstrated that circLARP4 acts as a tumorsuppressive factor and potential biomarker in gastric cancer. ${ }^{10}$ However, the roles of circLARP4 in NSCLC progression have not yet been explored. Recently, a novel regulatory mechanism has been proposed, in which circRNAs are capable of serving as competitive RNAs (ceRNAs) that sequester miRNAs via base-pair targeting to participate in cancer progression. ${ }^{11}$ Nevertheless, the detailed molecular basis of circLARP4 in NSCLC tumorigenesis stays still unclear.

Herein, we determined the reduced circLARP4 and increased miR-135b in NSCLC cells and tissues. Additionally, we found that circLARP4 served as a molecular sponge of miR-135b in NSCLC cells. Moreover, circLARP4 overexpression inhibited NSCLC development by targeting miR-135b through inactivation of the phosphatase and tension homolog (PTEN)/protein kinase B (AKT)/ hypoxia-inducible factor- $1 \alpha$ (HIF-1 $\alpha$ ) pathway. Thus, targeting circLARP4/miR-135b/PTEN/AKT/HIF-1 $\alpha$ pathway has potential to be effective for NSCLC.

\section{Materials and Methods}

\section{Clinical Samples}

A total of 20 NSCLC tissues and paired peritumoral healthy tissues that were verified by pathological examination were obtained from NSCLC patients undergoing surgical resection ranging from January 2017 to November 2018 at the Zhoukou Central Hospital (Zhoukou, China). These tissue samples were then promptly preserved at a $-80^{\circ} \mathrm{C}$ refrigerator for follow-up studies. All subjects enrolled in this study did not receive preoperative local or systemic therapy including chemotherapy or radiotherapy before the operation. This project was conducted in agreement with the Ethics Committee of Zhoukou Central Hospital and all involved patients were required to sign informed consent forms.

\section{Cell Culture and Transfection}

Human NSCLC cell lines (A549, H1299, H1975, and SPC-A-1), human normal lung epithelial cell line BEAS2B and human embryonic kidney cell line 293T were got from Shanghai Institute of Cell Biology, Chinese Academy of Sciences (Shanghai, China). These cells were fostered with $10 \%$ fetal bovine serum (FBS; PAN, Bavaria, Germany) supplemented RPMI-1640 medium (Boster, Wuhan, China) at $37^{\circ} \mathrm{C}$ with $5 \% \mathrm{CO}_{2}$.

PcDNA-circLARP4 (LARP4) and pcDNA control vector (pcDNA), siRNA targeting circLARP4 (si-LARP4) and its control (si-NC), lentiviral vector encoding circLARP4 (lenti-LARP4) and lentiviral vector (lenti-con), miR-135b mimics and a scrambled negative control (miR-NC), miR$135 \mathrm{~b}$ inhibitor (anti-miR-135b) and its negative control (anti-miR) were synthesized by GeneCopoeia (Rockville, MD, USA). The transfection concentration of miRNA mimics is $50 \mathrm{nM}$. When cells grew $70-80 \%$ confluence, the transfection into A549 and H1299 cells was carried out by means of the transfection reagent GeneJammer (Stratagene, Frampton, UK). The sequences of PcDNAcircLARP and si-LARP4 in cell transfections were exhibited in Supplementary File 1.

\section{Quantitative Real-Time PCR (qRT-PCR)}

RNAiso Plus (Takara Bio Inc., Japan) was adopted to isolate total RNA, followed by reverse-transcription into complementary DNA (cDNA) by the PrimeScript RT Reagent kit (Takara, Dalian, China). SYBR Premix EX Taq ${ }^{\mathrm{TM}}$ II kit (Takara) and miRNA qPCR Assay Kit (CWBIO, Beijing, China) were used to perform the subsequent qPCR analyses of circLARP4 and miR-135b expressions on a Quantstudio ${ }^{\mathrm{TM}}$ DX system 
(Applied Biosystems, Foster City, CA, USA), respectively. The $2^{-\Delta \Delta C T}$ method was adopted to calculate circLARP4 and miR-135b expressions, which were standardized by $\beta$-actin or $U 6$, respectively. The sequences of circLARP4 and miR-135b in qRT-PCR assay were shown in Supplementary File 1.

\section{Western Blot Analysis}

Total protein samples from tissues and cultured cells were isolated using ice-cold RIPA protein extraction buffer (ExCell Bio, Jiangsu, China). Protein samples $(25 \mu \mathrm{g}$ per lane) were resolved on 12\% SDS-PAGE at 80-120 V, followed by electro-transfer onto polyvinylidene difluoride (PVDF) membranes. After blockage of membranes in 5\% non-fat milk powder for $1 \mathrm{~h}$, primary antibodies including HK2 (Signalway Antibody, Pearland, TX, USA), PTEN (Abcam, Cambridge, MA, USA), AKT (Abcam), phosphorylated AKT (p-AKT) (Abcam), HIF-1 $\alpha$ (Abcam), and $\beta$-actin (Abcam) were taken to immune-stain the blots overnight at $4^{\circ} \mathrm{C}$. Thereafter, the blots were probed with the appropriate horseradish peroxidase (HRP)-linked secondary antibody (Biossci Biotechnology Co., Hubei, China). Subsequently, ECL Substrate (Pierce, Rockford, IL, USA) was utilized to visualize the protein bands.

\section{MTT Assay}

MTT assay was taken to examine cell proliferative ability. A549 and H1299 cells were introduced with pcDNA or LARP4 or together with miR-NC or miR-135b. At 0 h, 24 $\mathrm{h}, 48 \mathrm{~h}$ and $72 \mathrm{~h}$, cells were incubated with $20 \mu \mathrm{L}$ of MTT solution ( $5 \mathrm{mg} / \mathrm{mL}$, Sigma-Aldrich, St Louis, MO, USA), followed by incubation for further $4 \mathrm{~h}$. Thereafter, $150 \mu \mathrm{L}$ DMSO was added and a microplate reader (Molecular Devices, Silicon Valley, CA, USA) was adopted to read the absorbance under $490 \mathrm{~nm}$ wavelength.

\section{Transwell Invasion Assay}

Transwell chambers (Corning, Inc., Corning, New York, US) pre-coated with Matrigel were used to evaluate cell invasive ability. Briefly, a total of $2 \times 10^{4}$ transfected A549 and H1299 cells in $100 \mu \mathrm{L}$ serum-free RPMI-1640 medium was inoculated into the upper chamber and the lower chamber was filled with $500 \mu \mathrm{L}$ RPMI-1640 medium supplemented with $20 \%$ FBS. Following $24 \mathrm{~h}$ of incubation at $37^{\circ} \mathrm{C}$, the invaded cells on the upper surface of the chamber were carefully removed with a cotton swab. The invasive cells were fixed with $4 \%$ paraformaldehyde, stained with $0.1 \%$ crystal violet and imaged under an inverted light microscope (Nikon Corporation, Tokyo, Japan) at $\times 200$ magnification.

\section{Apoptosis Assay}

FITC Annexin V Apoptosis Detection Kit (Solarbio Life Sciences Co., Ltd., Beijing, China) was performed to detect the apoptosis rate of A549 and H1299 cells at $48 \mathrm{~h}$. Thereafter, a flow cytometer (FACSCalibur, Becton-Dickinson, San Jose, CA, USA) was used to measure the apoptotic rate.

\section{Measurement of Glucose Consumption and Lactate Production}

Following transfection, the A549 and H1299 cells were plated into 6-well plates and cultured for $48 \mathrm{~h}$. A glucose assay kit (BioVision, Milpitas, CA, USA) and a lactic acid assay kit (BioVision) were carried out to measure glucose consumption and lactate production in the supernatants of A549 and H1299 cells, respectively.

\section{Luciferase Reporter Assay}

Luciferase vectors pGL3-circLARP4-wild-type (circLARP4 (WT)) carrying the potential binding sites of miR-135b or pGL3-circLARP4-mutant (circLARP4 (MUT)) were obtained from GenePharma (Shanghai, China). 293T cells were plated into 96 well plates and cotransfected with the luciferase reporter plasmids and miR-135b, miR-NC, antimiR-135b, or anti-miR-NC using the transfection reagent GeneJammer (Stratagene). Forty-eight hours later, the Dual Luciferase Reporter Assay System Kit (Promega, Madison, WI, USA) was adopted to examine the luciferase activity.

\section{RNA Immunoprecipitation (RIP)}

The combination of circLARP4 and miR-135b was explored by RIP assay using the Magna RIP RNA-binding Protein Immunoprecipitation kit (Millipore). In brief, A549 cells were harvested and lysed with NP-40 lysis buffer containing cocktail (Roche Diagnostics, Basel, Switzerland). Thereafter, the supernatants were incubated at $4^{\circ} \mathrm{C}$ overnight with anti-human Argonaute 2 (Ago2) (Abcam) or negative control anti-IgG (Abcam) conjugated on magnetic beads in RIP buffer. Following this, the cold NT2 buffer was used to rinse the beads, followed by incubation with RNase-free DNase I (Promega) and Proteinase $\mathrm{K}$ with shaking to remove extra DNAs and proteins. Finally, the co-precipitated RNA was extracted and the relative enrichment of circLARP4 and miR-135b were examined using qRT-PCR. 


\section{Tumor Xenograft in vivo Assay}

The animal experiment procedures were executed with the approval of Animal Care and Use Committee of Zhoukou Central Hospital and followed the guidelines with consideration for laboratory animal welfare (Ministry of Science and Technology 2006). Briefly, BALB/C nude mice (female, 5-week-old, $20 \pm 2.2 \mathrm{~g}$ weight) were commercially purchased from the Slac Laboratory Animal Company (Shanghai, China) and maintained on a 12-h light-dark cycle in the pathogen-free condition. Approximately $5 \times 10^{7}$ lenti-LARP4- or lenti-contransducted A549 cells were subcutaneously implanted into the fossa axillaris of nude mice. One week later, tumor formation was monitored every week for 5 weeks and tumor volume was measured using the following formula: tumor volume $=$ $\left(\right.$ length $\times$ width $\left.^{2}\right) / 2$. After 5 weeks of injection, these mice were killed and the xenograft tumors were dissected, weighted and analyzed by qRT-PCR and Western blot.

\section{Statistics}

All data were displayed as mean \pm standard deviation (SD). Two-tailed Student's $t$-test with SPSS16.0 software (SPSS Inc., Chicago, IL, USA) was used to carry out statistical comparisons. $P<0.05$ was rendered statistically significant.

\section{Results}

\section{A Decrease of circLARP4 Expression Was Observed in NSCLC Tissues and Cells}

To clarify the roles of circLARP4 in NSCLC progression, we initially detected the circLARP4 expression level in 20 paired NSCLC tissue specimens and corresponding adjacent normal tissue samples by qRT-PCR. An aberrant downregulation of

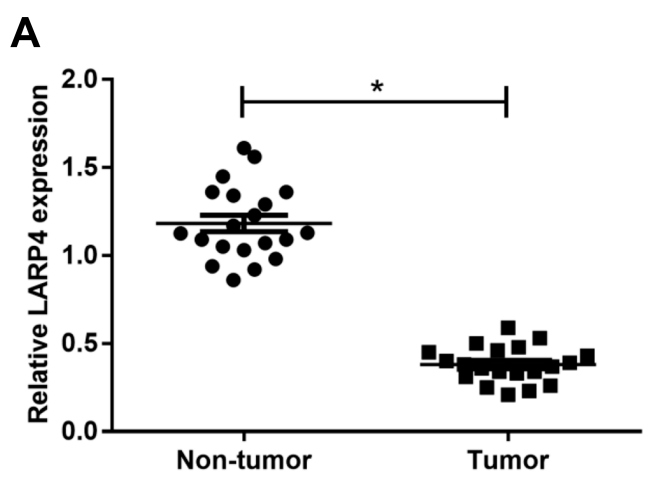

circLARP4 in NSCLC tissues was observed relative to that in adjacent normal tissues (Figure 1A). Meanwhile, we also detected circLARP4 expression in NSCLC cells and the results proved that circLARP4 expression was robustly decreased in NSCLC cell lines (A549, H1299, H1975, and SPC-A-1) relative to that immortalized human bronchial epithelial cell line BEAS-2B (Figure 1B). Moreover, especially in A549 and H1299, the most striking decreased expression in circLARP4 was observed. Thus, A549 and H1299 cell lines were chosen to study the roles of circLARP4 in NSCLC in vitro. These results suggested that circLARP4 expression was decreased in NSCLC tissues and cells.

\section{CircLARP4 Overexpression Inhibited the Progression of NSCLC Cells}

Considering the downregulation of circLARP4, we overexpressed circLARP4 to further analyze the biological function of circLARP4 by transfecting with LARP4 into A549 and H1299 cells. Transfection efficiency of LARP4 was verified by qRT-PCR and the results revealed that transfection with LARP4 significantly enhanced circLARP4 expression in A549 and H1299 cells in comparison to pcDNA-transfected group (Figure 2A). MTT assay presented that LARP4introduced A549 and H1299 cells exhibited a remarkable reduction of cell proliferation versus pcDNA-transfected cells (Figure 2B). Transwell invasion assay uncovered that cell invasive ability in A549 and H1299 cells was significantly dampened in response to circLARP4 overexpression versus control group (Figure 2C). Furthermore, flow cytometry analysis implied that the percentage of apoptotic A549 and H1299 cells was effectively increased following augmented expression of circLARP4 compared to control group (Figure 2D). Next, we explore the effect of forced expression of circLARP4

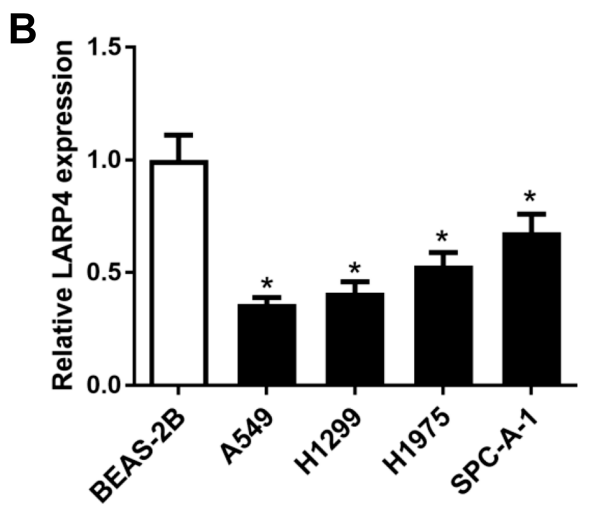

Figure I Expression pattern of circLARP4 in NSCLC clinical specimens and cells. (A) CircLARP4 expression pattern in 20 paired NSCLC tissue specimens and adjacent non-tumor tissue samples were detected by qRT-PCR. (B) CircLARP4 level in different cell lines (A549, HI299, HI975, SPC-A-I, and BEAS-2B) was examined by qRT-PCR. $* p<0.05$ compared with control. 

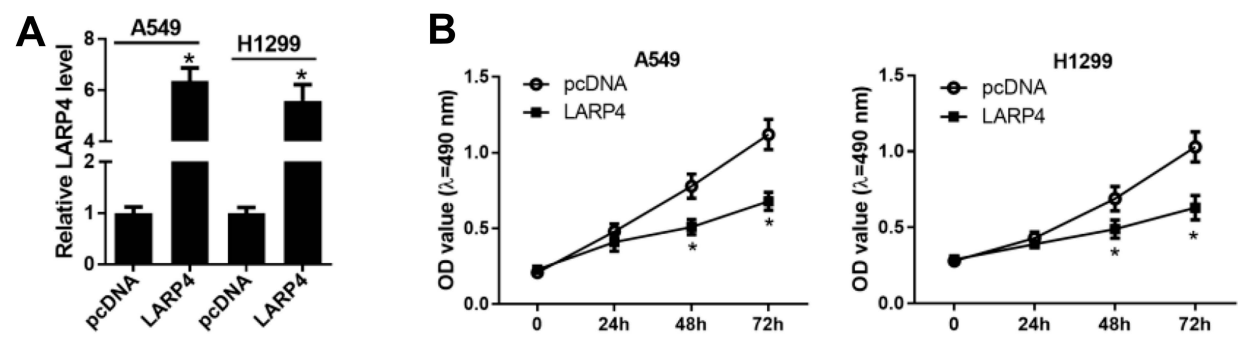

C
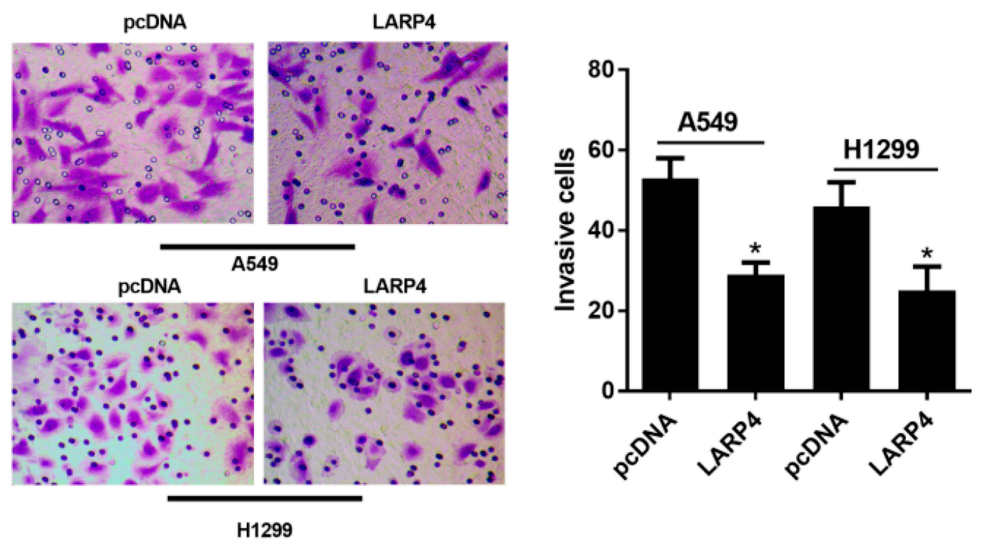

D
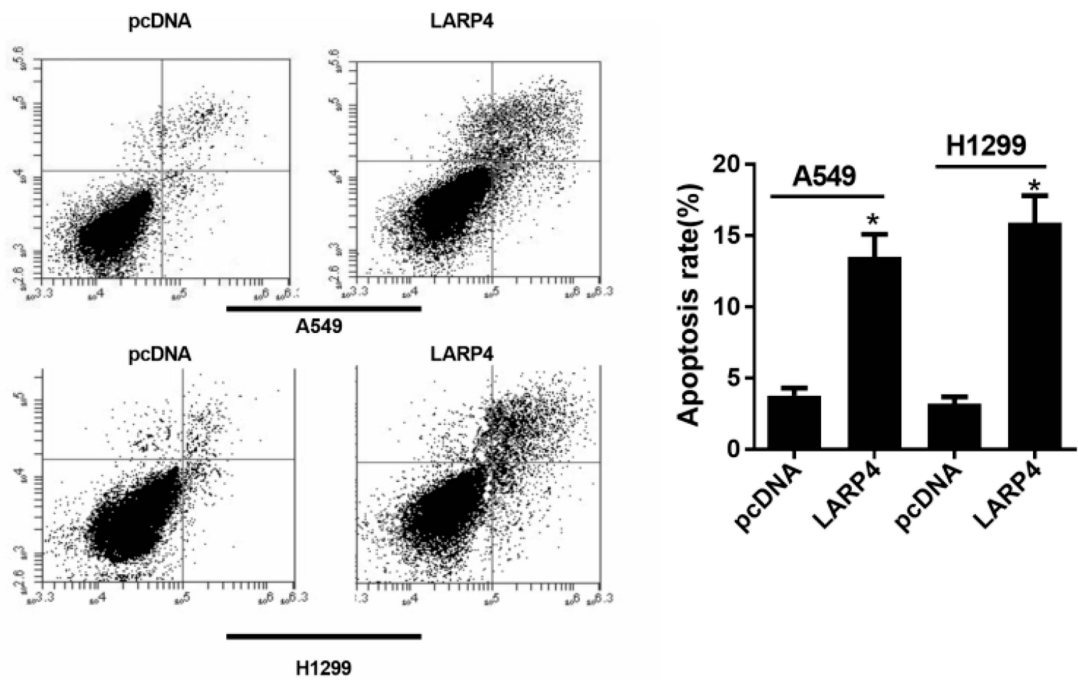

E

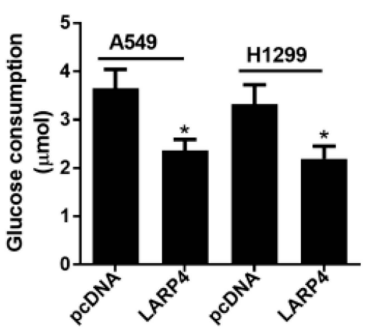

F

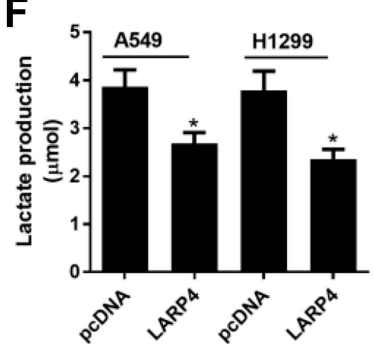

G

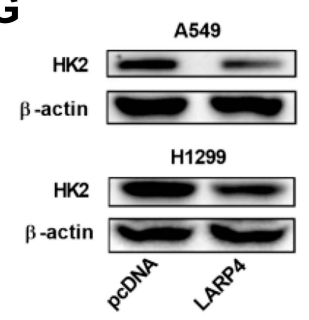

Figure 2 Effects of circLARP4 overexpression on NSCLC cell proliferation, invasion, glycolysis and apoptosis. (A) CircLARP4 expression in A549 and HI299 cells after delivery of LARP4 or pcDNA. (B) MTT assay for cell proliferation at $0 \mathrm{~h}, 24 \mathrm{~h}, 48 \mathrm{~h}$, and $72 \mathrm{~h}$ in A549 and HI299 cells received with LARP4 or pcDNA transfection. (C) Cell invasive ability was evaluated by transwell invasion assay after A549 and HI299 cells were delivered with LARP4 or pcDNA transfection. (D) Apoptosis in A549 and HI299 cells following delivery with LARP4 or pcDNA was evaluated by flow cytometry analysis. Glucose consumption (E) and lactate production (F) in A549 and HI299 cells after introduction with LARP4 or pcDNA. (G) Western blot analysis of HK2 protein level in LARP4 or pcDNA-transfected A549 and HI299 cells. *P < 0.05 compared with negative control. 
on glycolysis and the results showed that glucose consumption (Figure 2E) and lactate production (Figure 2F) were notably declined in LARP4-transfected A549 and H1299 cells when compared with that in pcDNA-transfected cells. Furthermore, we measured the expression of HK2, a critical mediator of aerobic glycolysis, in A549 and H1299 cells by Western blot. As compared with pcDNA group, ectopic expression of circLARP4 dramatically constrained HK2 protein level in A549 and H1299 cells (Figure 2G). These findings revealed that promotion of circLARP4 impeded the proliferation, invasion, glycolysis and promoted apoptosis of NSCLC cells.

\section{CircLARP4 Directly Interacted with miR-I35b in NSCLC Cells}

By searching for the potential downstream miRNAs of circLARP4 using Starbase3.0, we found that miR-135b was a possible binding candidate of circLARP4. Figure 3A showed the potential binding sites between circLARP4 and miR-135b. The results of luciferase reporter assay uncovered that introducing miR-135b significantly attenuated the luciferase activity of circLARP4 (WT), but there was no obvious change in the luciferase activity of circLARP4 (MUT) in 293T cells (Figure 3B). Reciprocally, miR-135b inhibition dramatically elevated the luciferase activity of circLARP4 (WT) rather than circLARP4 (MUT) in 293T cells (Figure 3B). Additionally, RIP assay further demonstrated that circLARP4 and
miR-135b were preferentially enriched among Ago2 immunoprecipitates in A549 cell lysates (Figure 3C), indicating that circLARP4 directly interacted with miR-135b. A marked upregulation of miR-135b was observed in NSCLC tissues and NSCLC cell lines (Figure 3D and E). Furthermore, circLARP4 expression was distinctly elevated upon LARP4 transfection and markedly decreased following si-LARP4 introduction in A549 cells (Figure 3F). LARP4overxpressing A549 cells exhibited an evident reduction of miR-135b expression and LARP4-silencing A549 cells showed a substantial augment of miR-135b expression in A549 cells (Figure 3G). Together, these results suggested that miR-135b direct interacting with circLARP4 in NSCLC cells was demonstrated.

\section{miR-135b Partially Abolished circLARP4-Mediated Tumor-Suppressing Effects on NSCLC Cells}

Next, aiming at exploring whether miR-135b was involved in the tumor-suppressing effects of circLARP4 on NSCLC cells, we carried out rescue experiments by delivering with LARP4, pcDNA, LARP4 + miR-135b or LARP4 + miRNC into A549 and H1299 cells. MTT assay hinted that the inhibitory effect of LARP4 on cell viability was partially ameliorated following the promotion of miR-135b in A549 and H1299 cells (Figure 4A). As demonstrated by
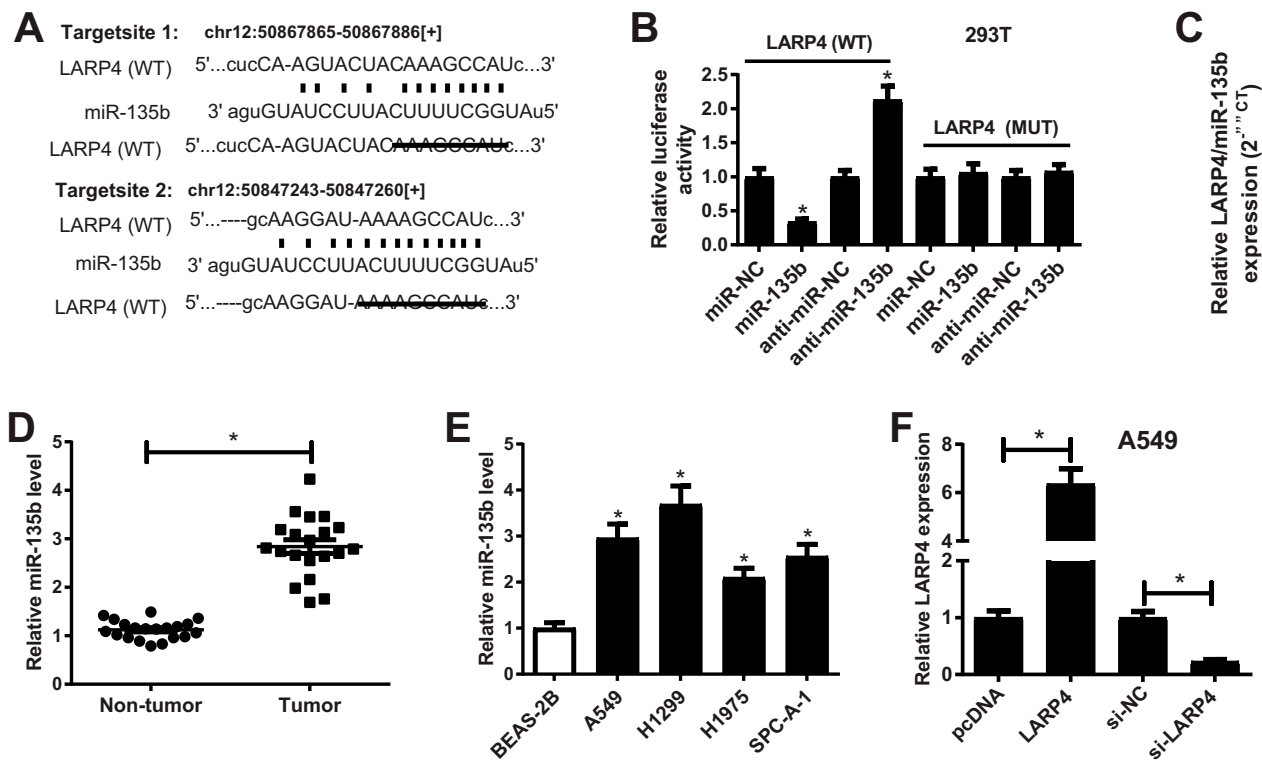

Figure 3 The association between circLARP4 and miR-I35b in NSCLC cells. (A) Sequence alignment of miR-I35b with binding sites in wild-type and mutant-type regions of circLARP4 was presented. The interaction between circLARP4 and miR-I35b was investigated by luciferase reporter assay (B) and RIP assay (C). (D) qRT-PCR analysis of miR-I35b expression in NSCLC tissues and corresponding adjacent normal tissue samples. (E) qRT-PCR analysis of miR-I35b expression in different cell lines (A549, HI299, HI975, SPC-A-I, and BEAS-2B). (F and G) A549 cells were received with LARP4, si-LARP4, or respective controls transfection, followed by detection of circLARP4 and miR-135b expressions by qRT-PCR. *P $<0.05$ compared with negative control. 
A

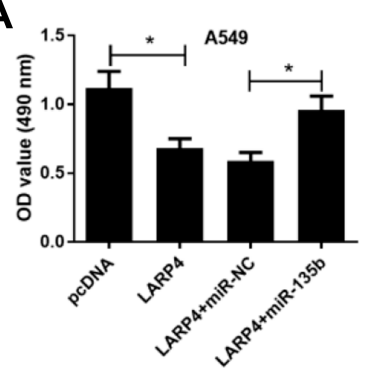

C

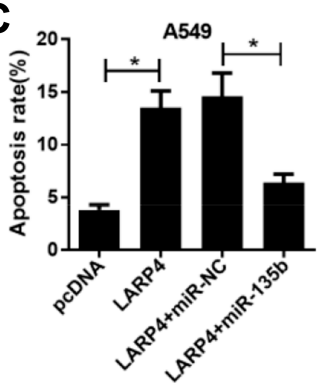

E

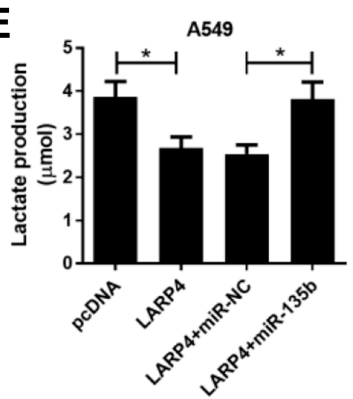

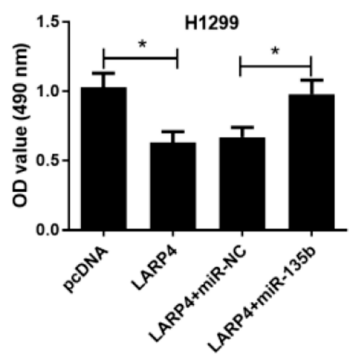
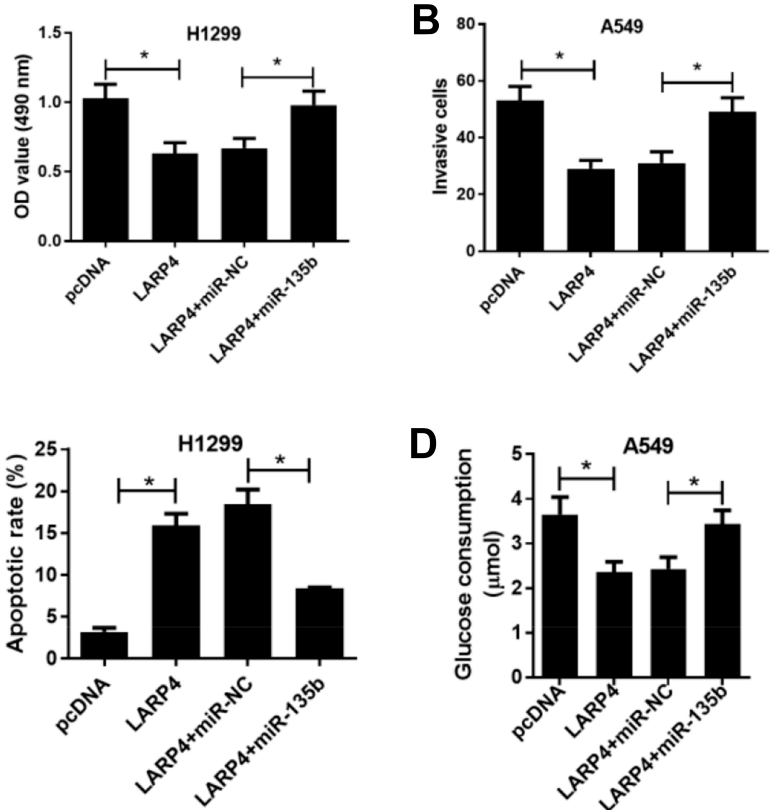

D

$\mathbf{F}$

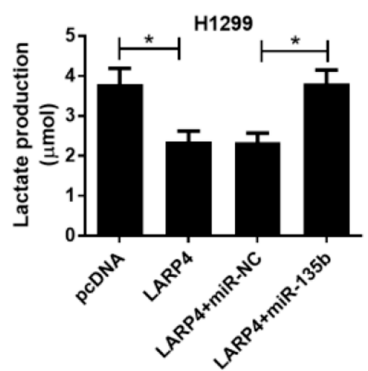

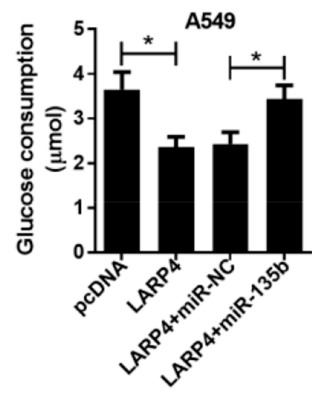
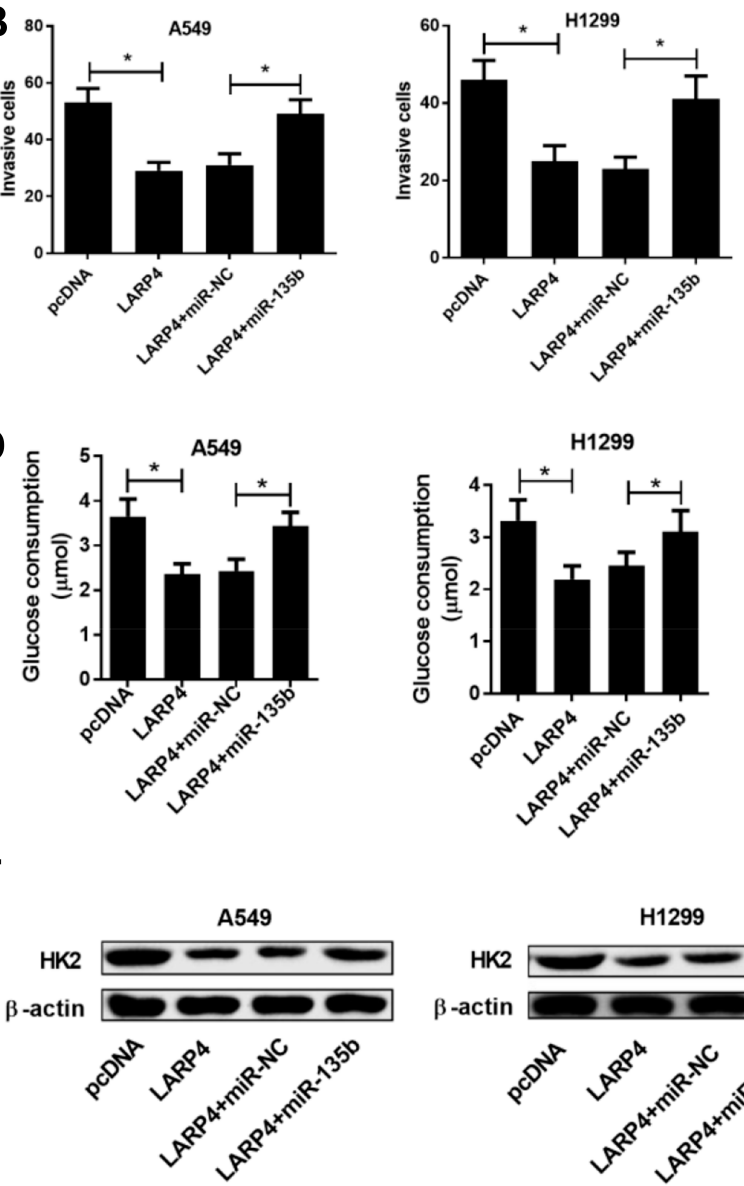

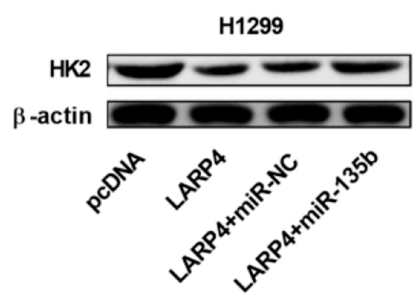

Figure 4 Effects of circLARP4 or together with miR-I35b on NSCLC cell viability, glycolysis and apoptosis. A549 and HI299 cells were delivered with LARP4, pcDNA, LARP4 + miR-135b or LARP4 + miR-NC. (A) Cell viability of treated A549 and HI 299 cells was detected by MTT assay. (B) Cell invasion potential was assessed by transwell invasion assay. (C) The percentage of apoptotic cells in the transfected A549 and HI299 cells was detected by flow cytometry analysis. (D and E) Glucose consumption and lactate production in the introduced A549 and HI299 cells. (F) HK2 protein level in the transfected A549 and HI 299 cells was measured by Western blot. *P < 0.05 compared with negative control.

transwell invasion assay, robust expression of miR-135b strikingly rescued LARP4-induced reduction of cell invasion in A549 and H1299 cells (Figure 4B). In addition, miR-135b greatly counteracted restoring expression of circLARP4-induced apoptosis in A549 and H1299 cells (Figure 4C). As shown in (Figure 4D and E), enforced expression of miR-135b dramatically overturned the effects of circLARP4 overexpression in increasing glucose consumption and lactate production in A549 and H1299 cells. Meanwhile, HK2 protein level was remarkably decreased in LARP4-transfected A549 and H1299 cells, while was successfully abolished in response to miR-135b overexpression (Figure 4F). Together, we concluded that circLARP4 overexpression regulated cell proliferation, invasion, glycolysis and promoted apoptosis of NSCLC cells by downregulating miR-135b.

\section{CircLARP4 Overexpression Inhibited} NSCLC Xenograft Tumor Formation and Decreased miR-135b Expression

Next, to verify the in vivo effect of circLARP4 on NSCLC xenograft development, a xenograft tumor model was established and employed. Tumor formation was monitored weekly for 5 weeks. After 5 weeks of implantation, the xenograft tumors were dissected. CircLARP4 expression was potently boosted (Figure $5 \mathrm{~A}$ ) and miR-135b expression was conspicuously repressed (Figure 5B) in the resected tumor tissues from lenti-LARP4 group relative to that in lenti-con group. Moreover, it was indicated that regained expression of circLARP4 substantially retarded tumor growth and weight when compared to control group (Figure 5C and D). Together, we concluded 
that circLARP4 overexpression inhibited NSCLC tumorigenesis in vivo by decreasing miR-135b expression.

\section{CircLARP4 Overexpression Inhibited the PTEN/AKT/HIF-I $\alpha$ Signaling Pathway via Downregulating miR-I35b in NSCLC in vitro and in vivo}

As shown in (Figure 6A and B), robust expression of circLARP4 significantly augmented PTEN expression and notably restricted the protein levels of p-AKT and HIF- $1 \alpha$, but did not affect AKT expression in A549 cells and xenograft tumor tissues from lenti-LARP4 group, suggesting that circLARP4 overexpression inhibited the PTEN/AKT/ HIF-1 $\alpha$ signaling pathway in NSCLC in vitro and in vivo. However, forced expression of miR-135b dramatically reversed the inhibition of the PTEN/AKT/HIF-1 $\alpha$ signaling pathway in A549 cells induced by circLARP4 overexpression (Figure 6A). These results demonstrated that circLARP4 overexpression inhibited the PTEN/AKT/HIF$1 \alpha$ signaling pathway via downregulating miR-135b in NSCLC in vitro and in vivo.

\section{Discussion}

In the last few years, increasing attention has received concerning the functions of circRNAs in cancer progression. ${ }^{12}$ Increasing experimental data have shown that aberrant expression of circRNAs is reportedly associated with the oncogenesis and development of malignant tumors and circRNAs are thus regarded as diagnostic biomarkers and compelling therapeutic targets for multiple cancers, including NSCLC. ${ }^{13,14}$ For example, an upregulation of circZFR has been observed in NSCLC tissues and cells, and knockdown of circZFR inhibits cell proliferation, migration and invasion of NSCLC cells by acting as a miR-101-3p sponge to enhance cullin 4B (CUL4B) expression. ${ }^{15}$ Circ_0020123 has been reported to be highly expressed in NSCLC and its silencing hinders cell growth, migration, invasion and enhances apoptosis in NSCLC cells by sponging miR-488$3 p$ to release its inhibition on a disintegrin and metalloproteinase 9 (ADAM9) expression. ${ }^{16}$ CircVANGL1 has been demonstrated to be ectopically expressed in NSCLC tissues and cells, and functions as an oncogene to promote NSCLC progression partly through miR-195/Bcl-2 axis. ${ }^{17}$ CircLARP4, a circRNA deriving from LARP4 gene locus, has been revealed to be downregulated and serve as a potential biomarker in ovarian cancer prognosis, ${ }^{18}$ gastric cancer, ${ }^{10}$ and hepatocellular carcinoma. ${ }^{19}$ Moreover, the tumor-suppressive roles of circLARP4 in gastric cancer ${ }^{10}$ and hepatocellular carcinoma ${ }^{19}$ have been also well documented. However, the expression and functional roles of circLARP4 in NSCLC have not yet been elucidated. In this research, a low expression of circLARP4 was found in NSCLC tissues and cells. Moreover, further experiments uncovered that ectopic expression of circLARP4 dampened cell proliferation, invasion and glycolysis, and facilitated apoptosis in NSCLC cells in vitro, as well as restricted NSCLC xenograft tumor formation in vivo. Together, we demonstrated that circLARP4 exerted tumor-suppressing effect on NSCLC.

In contrast to normal cells, most proliferative malignant tumor cells preferentially depend on aerobic glycolysis instead of oxidative phosphorylation for energy production during malignant progression, which is defined as "the Warburg effect". ${ }^{20}$ As a well-recognized characteristic of cancer, aerobic glycolysis, characterized by enhanced glucose consumption and lactate production, is frequently activated in a variety of cancers and contributes to tumor growth and development. ${ }^{21} \mathrm{HK}$, a rate-limiting enzyme which catalyzes the first committed step of glycolysis, is well recognized to contribute to aerobic glycolysis. ${ }^{22}$ A significant upregulation of $\mathrm{HK} 2$ has been observed in various malignant tumors and is correlated with poor prognosis. ${ }^{22}$ Substantive studies have demonstrated that the circRNAs function as crucial regulators of cancer metabolism, particularly in the Warburg effect. ${ }^{23}$ For example, circDENND4C knockdown decreased glycolysis, migration and invasion in breast cancer cells under hypoxia. ${ }^{24}$ In oral squamous cell carcinoma cells, cell proliferation and glycolysis were remarkably downregulated by circRNA_100290 silence. ${ }^{25}$ In this research, we proved the inhibition of glycolysis by circLARP4 overexpression in NSCLC cells, as evidenced by the reduced HK2 level, glucose consumption and lactate production in NSCLC cells.

As a novel regulatory mechanism, circRNAs serve as miRNA sponges to competitively inhibit miRNA expressions and functions in multiple cancers. ${ }^{26}$ For instance, $\mathrm{Li}$ et al have found that an upregulation of circRNA_102958 in colorectal cancer (CRC) is observed and CRC cell growth, migration and invasion are retarded due to circRNA_102958 knockdown-induced upregulation of miR-585. ${ }^{27}$ Zheng et al have reported that circPCMTD1 contributes to the promotion of glioma progression by serving as a sponge of miR$224-5 p{ }^{28}$ In this research, bioinformatics analyses showed that miR-135b shared complementary binding with the 3'UTR of circLARP4. Compelling evidence has suggested 
A

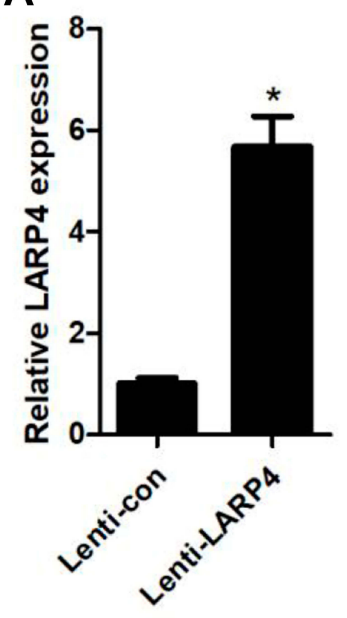

C
B

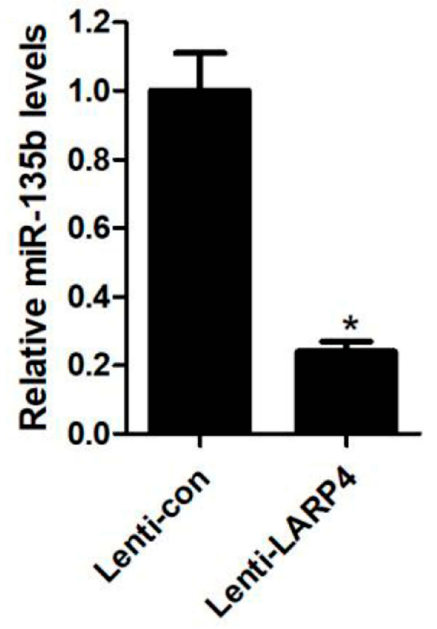

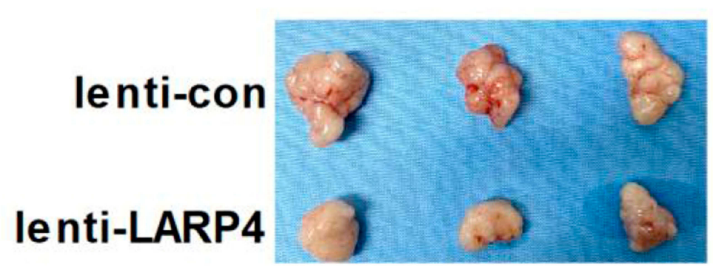
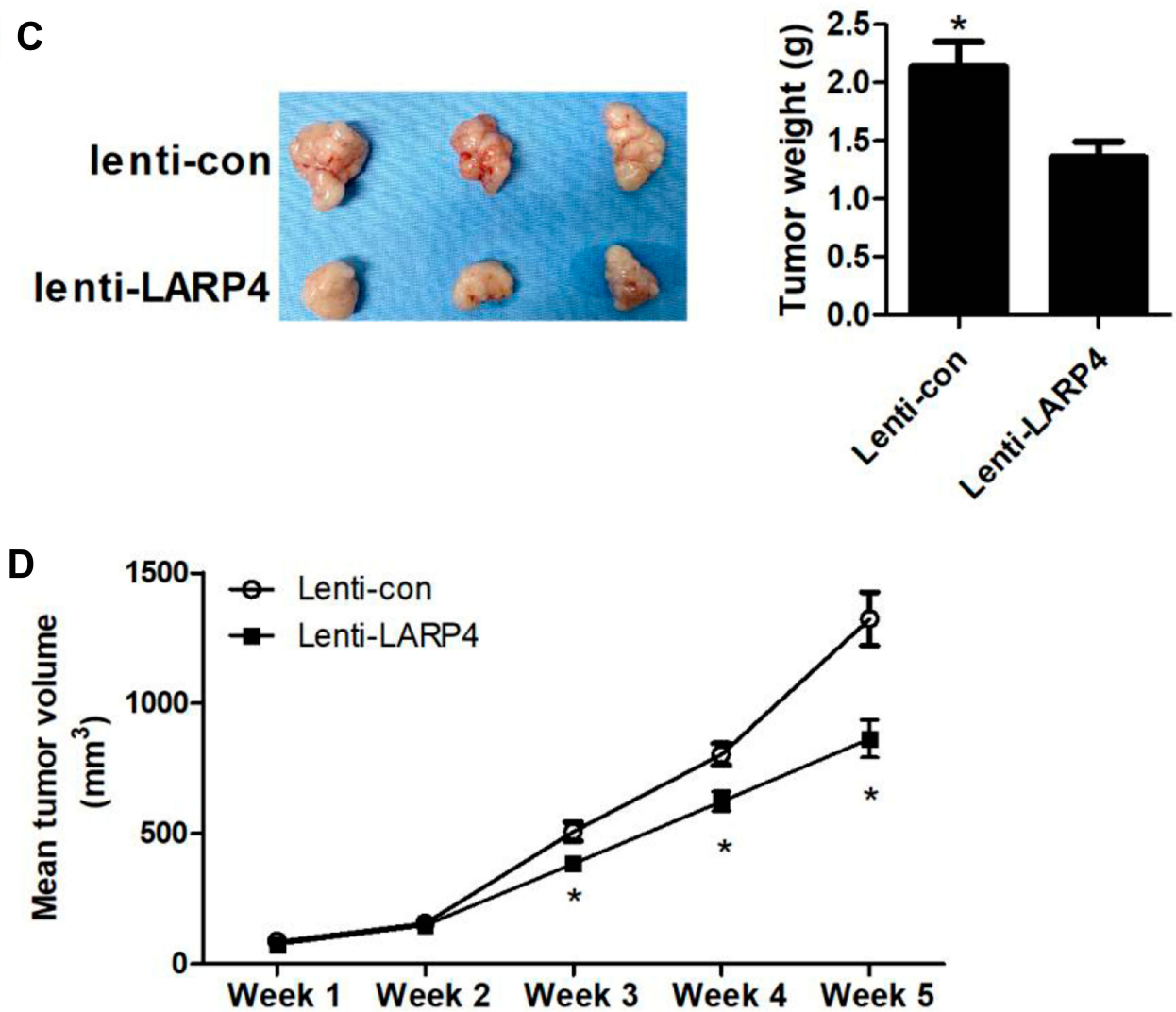

Figure 5 Effects of circLARP4 on NSCLC tumor growth and miR-135b expression in vivo. (A and B) CircLARP4 and miR-I35b expressions in the xenograft tumors in nude mice derived from subcutaneous injection of A549 cells transfecting with lenti-LARP4 or lenti-con were estimated by qRT-PCR. (C) After 5 weeks of implantation, the xenograft tumors were dissected and weighed. (D) Tumor formation was monitored weekly for 5 weeks. $* P<0.05$ compared with negative control.

that the roles of miR-135b in different types of cancer are contradictory. For example, it is reported that miR-135b acts as a tumor-suppressor factor in prostate cancer ${ }^{29}$ and osteoblastoma. $^{30}$ However, miR-135b was believed as a tumor-promoting miRNA in various malignancies, such as cervical cancer, ${ }^{31}$ breast cancer $^{32}$ as well as NSCLC. ${ }^{33}$
Following validation, we demonstrated that circLARP4 acted as a ceRNA to suppress miR-135b expression in NSCLC in vitro and in vivo. Our rescue experiments demonstrated that miR-135b partially abolished the suppressive effects on cell viability, invasion and glycolysis, and facilitative effects on apoptosis in NSCLC cells mediated by 
A
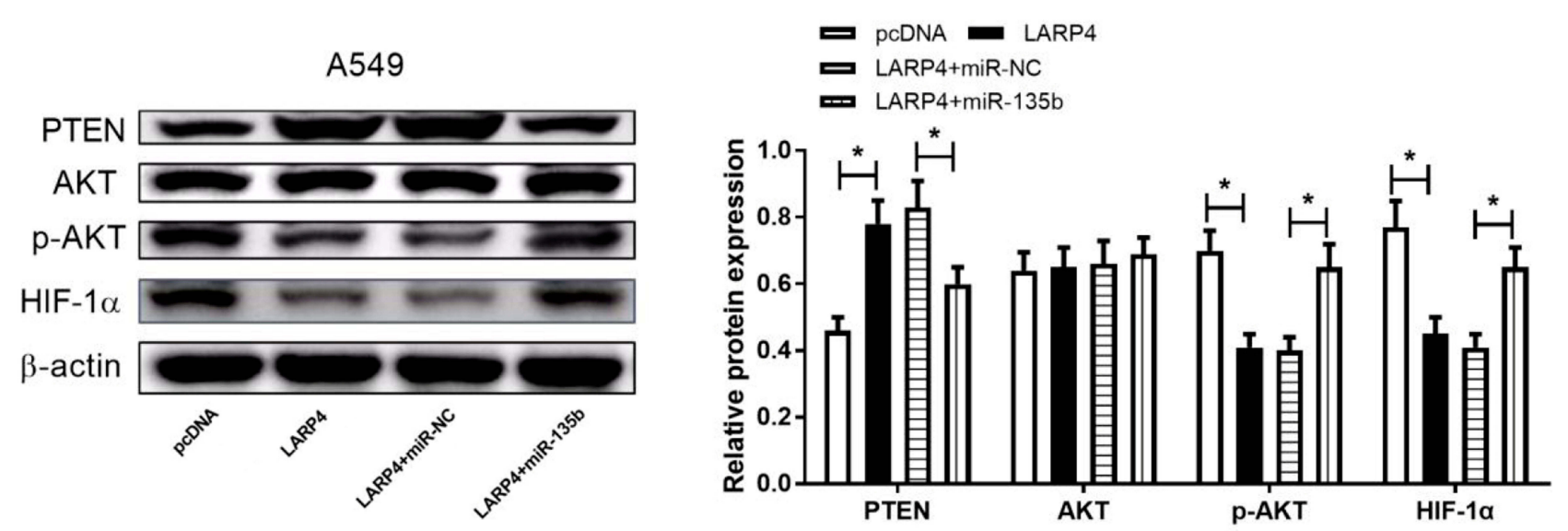

B
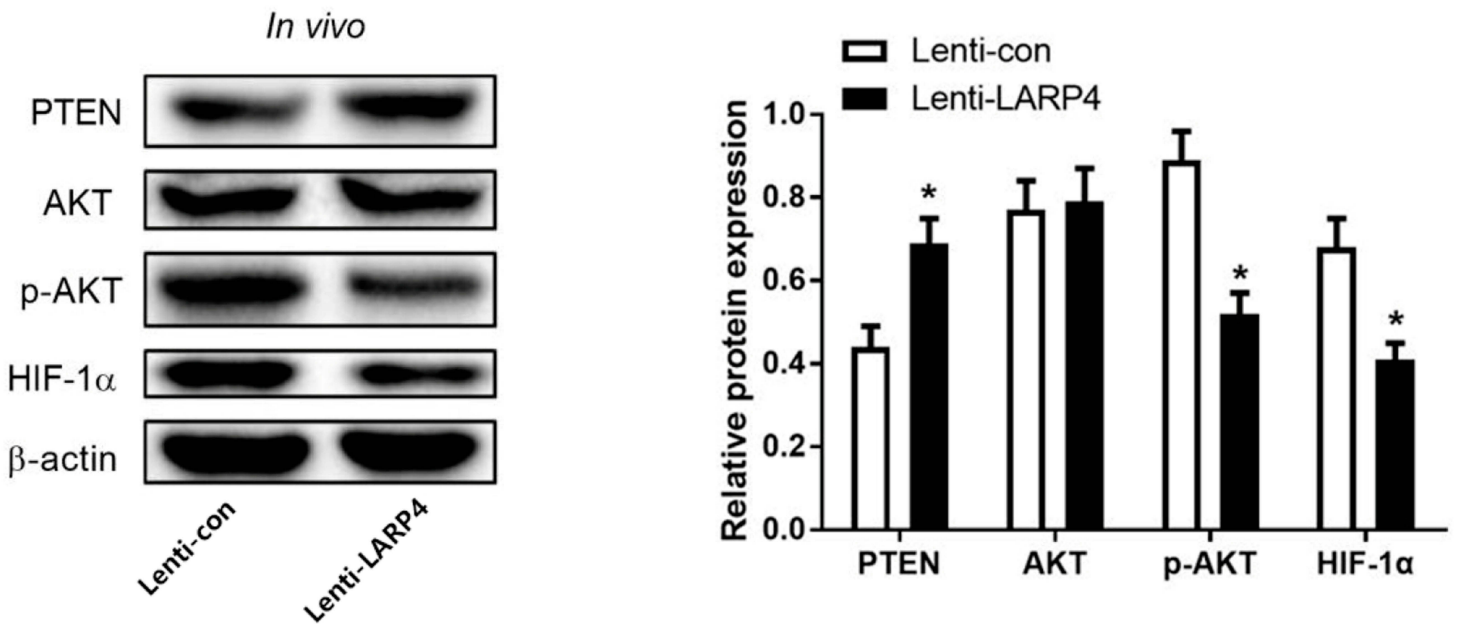

Figure 6 Effects of circLARP4 or along with miR-I35b on the PTEN/AKT/HIF- I $\alpha$ signaling pathway in NSCLC. (A) The protein levels of PTEN, $\mathrm{P}-\mathrm{AKT}$, AKT and HIF-I $\alpha$ in A549 cells transfected with LARP4, pcDNA, LARP4 + miR-I35b or LARP4 + miR-NC were detected by Western blot analysis. (B) Western blot analysis of PTEN, p-AKT, AKT and HIF-I $\alpha$ protein levels in xenograft tumor tissues. $* P<0.05$.

circLARP4, suggesting that circLARP4 suppressed the progression of NSCLC by sponging miR-135b.

PTEN, a well-known tumor suppressor, is considered as an antagonist of the actions of Akt pathway in various cancers. ${ }^{34}$ A decrease of PTEN expression and activity occurs frequently observed in many tumors including NSCLC, which is associated with accelerated NSCLC growth and invasion. ${ }^{35}$ It is commonly reckoned that the PTEN/Akt pathway is frequently constitutively activated in various types of human tumors including NSCLC and participates in the regulation of various biological processes in cancer cells, including cell growth, invasion, glycolysis and tumorigenesis. ${ }^{36}$ Therefore, inhibiting the activation of the PTEN/AKT pathway may be a therapeutic approach for NSCLC. ${ }^{37}$ HIF-1 $\alpha$, a transcriptional factor that is overexpressed in cancer cells, is identified as a key regulator of aerobic gycolysis and tumorigenicity in cancer cells. ${ }^{38}$ Moreover, emerging evidence shows that the AKT pathway plays a critical role in controlling HIF- $1 \alpha$ expression in multiple human tumors. ${ }^{39}$ In our study, we demonstrated an increase of PTEN expression and an inhibition of AKT phosphorylation and HIF- $1 \alpha$ expression by circLARP4 overexpression in NSCLC cells and xenograft tumors, which were abolished following reintroduction with miR-135b, suggesting that circLARP4 overexpression inhibited the PTEN/AKT/HIF-1 $\alpha$ signaling pathway via downregulating miR-135b in NSCLC in vitro and in vivo. Considering the roles of PTEN/ AKT/HIF-1 $\alpha$ signaling pathway in the progression of NSCLC, we concluded that circRNA LARP4 suppressed the growth and progression in NSCLC by sponging miR-135b via inhibiting the PTEN/AKT/HIF-1 $\alpha$ signaling pathway. 


\section{Conclusion}

Taken together, we discovered a low expression of circLARP4 in NSCLC tissues and cells. Promotion of circLARP4 retarded cell proliferation, invasion and glycolysis, and facilitated apoptosis in NSCLC cells in vitro, as well as impeded NSCLC tumor formation in vivo via interacting with miR-135b through inactivation of the PTEN/AKT/HIF$1 \alpha$ signaling pathway, which broadens our understanding concerning the role of circLARP4 in NSCLC carcinogenesis. We identified crosstalk between circLARP4 and miR$135 \mathrm{~b}$, providing a novel therapeutic candidate for NSCLC.

However, limitations exist in our study. Herein, total of 20 paired NSCLC patients were enrolled and the expression profile of circLARP4 was preliminarily detected. Because the current sample size is too small, the relationship between molecular expression and clinical data cannot be analyzed and the survival data are not available now. Moreover, it is needed to explore whether siLARP4 affects cell proliferation, invasion and apoptosis.

\section{Disclosure}

The authors have no conflicts of interest in this work.

\section{References}

1. Siegel RL, Miller KD, Jemal A. Cancer statistics. CA Cancer J Clin. 2017;67(1):7-30. doi:10.3322/caac.21387

2. Ettinger DS, Akerley W, Bepler G, et al. Non-small cell lung cancer. $J$ Natl Compr Canc Netw. 2010;8(7):740-801. doi:10.6004/jncen. 2010.0056

3. Chen Z, Fillmore CM, Hammerman PS, et al. Non-small-cell lung cancers: a heterogeneous set of diseases. Nat Rev Cancer. 2014;14 (8):535-546. doi:10.1038/nrc3775

4. Hirsch FR, Scagliotti GV, Mulshine JL, et al. Lung cancer: current therapies and new targeted treatments. Lancet. 2017;389 (10066):299-311. doi:10.1016/S0140-6736(16)30958-8

5. Wilusz JE, Sharp PA. Molecular biology. A circuitous route to noncoding RNA. Science. 2013;340(6131):440-441. doi:10.1126/ science. 1238522

6. Wilusz JE. A 360 degrees view of circular RNAs: from biogenesis to functions. Wiley Interdiscip Rev RNA. 2018;9(4):e1478. doi:10.1002/ wrna. 1478

7. Patop IL, Kadener S. circRNAs in Cancer. Curr Opin Genet Dev. 2018;48:121-127. doi:10.1016/j.gde.2017.11.007

8. Hu W, Bi ZY, Chen ZL, et al. Emerging landscape of circular RNAs in lung cancer. Cancer Lett. 2018;427:18-27. doi:10.1016/j. canlet.2018.04.006

9. Seetharaman S, Flemyng E, Shen J, et al. The RNA-binding protein LARP4 regulates cancer cell migration and invasion. Cytoskeleton. 2016;73(11):680-690. doi:10.1002/cm.21336

10. Zhang J, Liu H, Hou L, et al. Circular RNA_LARP4 inhibits cell proliferation and invasion of gastric cancer by sponging miR-424-5p and regulating LATS1 expression. Mol Cancer. 2017;16(1):151. doi:10.1186/s12943-017-0719-3

11. Zhong Y, Du Y, Yang X, et al. Circular RNAs function as ceRNAs to regulate and control human cancer progression. Mol Cancer. 2018;17 (1):79. doi:10.1186/s12943-018-0827-8
12. Zhu LP, He YJ, Hou JC, et al. The role of circRNAs in cancers. Biosci Rep. 2017;37(5):5. doi:10.1042/BSR20170750

13. Meng S, Zhou H, Feng Z, et al. CircRNA: functions and properties of a novel potential biomarker for cancer. Mol Cancer. 2017;16(1):94. doi:10.1186/s12943-017-0663-2

14. Chen L, Nan A, Zhang N, et al. Circular RNA 100146 functions as an oncogene through direct binding to miR-361-3p and miR-615-5p in non-small cell lung cancer. Mol Cancer. 2019;18(1):13. doi:10.1186/ s12943-019-0943-0

15. Zhang H, Wang X, Hu B, et al. Circular RNA ZFR accelerates non-small cell lung cancer progression by acting as a miR-101-3p sponge to enhance CUL4B expression. Artif Cells Nanomed Biotechnol. 2019;47 (1):3410-3416. doi:10.1080/21691401.2019.1652623

16. Wan J, Hao L, Zheng X, et al. Circular RNA circ_0020123 promotes non-small cell lung cancer progression by acting as a ceRNA for miR-488-3p to regulate ADAM9 expression. Biochem Biophys Res Commun. 2019;515(2):303-309. doi:10.1016/j.bbrc.2019.05.158

17. Wang L, Ma H, Kong W, et al. Up-regulated circular RNA VANGL1 contributes to progression of non-small cell lung cancer through inhibition of miR-195 and activation of Bcl-2. Biosci rep. 2019;39(6).

18. Zou T, Wang PL, Gao Y, et al. Circular RNA_LARP4 is lower expressed and serves as a potential biomarker of ovarian cancer prognosis. Eur Rev Med Pharmacol Sci. 2018;22(21):7178-7182. doi:10.26355/eurrev_201811_16250

19. Chen Z, Zuo X, Pu L, et al. circLARP4 induces cellular senescence through regulating miR-761/RUNX3/p53/p21 signaling in hepatocellular carcinoma. Cancer sci. 2019;110(2):568-581.

20. Liberti MV, Locasale JW. The warburg effect: how does it benefit cancer cells? Trends Biochem Sci. 2016;41(3):211-218. doi:10.1016/ j.tibs.2015.12.001

21. Diaz-Ruiz R, Rigoulet M, Devin A. The warburg and crabtree effects: on the origin of cancer cell energy metabolism and of yeast glucose repression. Biochim Biophys Acta. 2011;1807(6):568-576. doi:10.1016/j.bbabio.2010.08.010

22. Lincet H, Icard P. How do glycolytic enzymes favour cancer cell proliferation by nonmetabolic functions? Oncogene. 2015;34 (29):3751-3759. doi:10.1038/onc.2014.320

23. Yu T, Wang Y, Fan Y, et al. CircRNAs in cancer metabolism: a review. J hematol oncol. 2019;12(1):90.

24. Ren S, Liu J, Feng Y, et al. Knockdown of circDENND4C inhibits glycolysis, migration and invasion by up-regulating $\mathrm{miR}-200 \mathrm{~b} / \mathrm{c}$ in breast cancer under hypoxia. J Exp Clin Cancer Res. 2019;38(1):388. doi:10.1186/s13046-019-1398-2

25. Chen X, Yu J, Tian H, et al. Circle RNA hsa_circRNA_100290 serves as a ceRNA for miR-378a to regulate oral squamous cell carcinoma cells growth via glucose transporter-1 (GLUT1) and glycolysis. J Cell Physiol. 2019;234(11):19130-19140. doi:10.1002/jcp.28692

26. Shang Q, Yang Z, Jia R, et al. The novel roles of circRNAs in human cancer. Mol Cancer. 2019;18(1):6. doi:10.1186/s12943-018-0934-6

27. Li R, Wu B, Xia J, et al. Circular RNA hsa_circRNA_102958 promotes tumorigenesis of colorectal cancer via miR-585/CDC25B axis. Cancer Manag Res. 2019;11:6887-6893. doi:10.2147/CMAR. S212180

28. Zheng SQ, Qi Y, Wu J, et al. CircPCMTD1 acts as the sponge of miR-224-5p to promote glioma progression. Front Oncol. 2019;9:398. doi:10.3389/fonc. 2019.00398

29. Wang N, Tao L, Zhong H, et al. miR-135b inhibits tumour metastasis in prostate cancer by targeting STAT6. Oncol Lett. 2016;11 (1):543-550. doi:10.3892/ol.2015.3970

30. Li ZW, Zhu YR, Zhou XZ, et al. microRNA-135b expression silences Ppm1e to provoke AMPK activation and inhibit osteoblastoma cell proliferation. Oncotarget. 2017;8(16):26424-26433. doi:10.18632/ oncotarget. 15477

31. Xu Y, Zhao S, Cui M, et al. Down-regulation of microRNA-135b inhibited growth of cervical cancer cells by targeting FOXO1. Int J Clin Exp Pathol. 2015;8(9):10294-10304. 
32. Lv ZD, Xin HN, Yang ZC. miR-135b promotes proliferation and metastasis by targeting APC in triple-negative breast cancer. J cellular physiol. 2019;234(7):10819-10826.

33. Lin CW, Chang YL, Chang YC, et al. MicroRNA-135b promotes lung cancer metastasis by regulating multiple targets in the hippo pathway and LZTS1. Nat Commun. 2013;4(1):1877. doi:10.1038/ncomms2876

34. Salmena L, Carracedo A, Pandolfi PP. A Carracedo, and P P Pandolfi. Tenets of PTEN tumor suppression. Cell. 2008;133(3):403-414. doi:10.1016/j.cell.2008.04.013

35. Soria JC, Lee HY, Lee JI, et al. Lack of PTEN expression in non-small cell lung cancer could be related to promoter methylation. Clin Cancer Res. 2002;8(5):1178-1184.

36. Hong SY, Yu FX, Luo Y, et al. Oncogenic activation of the PI3K/Akt pathway promotes cellular glucose uptake by downregulating the expression of thioredoxin-interacting protein. Cell Signal. 2016;28 (5):377-383. doi:10.1016/j.cellsig.2016.01.011
37. Matsuda S, Nakagawa Y, Kitagishi Y, et al. Reactive oxygen species, superoxide dismutases, and PTEN-p53-AKT-MDM2 signaling loop network in mesenchymal stem/stromal cells regulation. Cells. 2018;7 (5):36. doi:10.3390/cells 7050036

38. Singh D, Arora R, Kaur P, et al. Overexpression of hypoxia-inducible factor and metabolic pathways: possible targets of cancer. Cell Biosci. 2017;7(1):62. doi:10.1186/s13578-017-0190-2

39. Pore N, Jiang Z, Shu HK, et al. Akt1 activation can augment hypoxia-inducible factor-1alpha expression by increasing protein translation through a mammalian target of rapamycin-independent pathway. Mol Cancer Res. 2006;4(7):471-479. doi:10.1158/15417786.MCR-05-0234

\section{Publish your work in this journal}

OncoTargets and Therapy is an international, peer-reviewed, open access journal focusing on the pathological basis of all cancers, potential targets for therapy and treatment protocols employed to improve the management of cancer patients. The journal also focuses on the impact of management programs and new therapeutic agents and protocols on patient perspectives such as quality of life, adherence and satisfaction. The manuscript management system is completely online and includes a very quick and fair peer-review system, which is all easy to use. Visit http://www.dovepress.com/ testimonials.php to read real quotes from published authors. 